\title{
The Protective Role of Vitamin C against Formaldehyde induced- hepatotoxicity and nephrotoxicity in Male Rats
}

\author{
Shang Z. Abdulqader ${ }^{1}$, Inaam A.Mustafa ${ }^{2}$ \\ ${ }^{1,2}$ (Department of Biology, College of Science/ University of Salaahaddin/Iraq)
}

\begin{abstract}
Formaldehyde was administrated by gavage into male albino rats for 7 day as a single dose (1ml), and the histological changes in liver and kidney were investigated using paraffin method. This compound has caused hepatotoxicity which included degeneration of the hepatocytes, and appearing of inflammatory leucocytes. This compound has also caused nephrotoxicity which included high inflammation degree and degenerative effect on kidney tubule cells. When given to the formalin exposed rats, vitamin C caused recovery of the changes in the liver and kidney, i.e. it has been succeeded in playing a protective role against formalin hepatotoxicity and nephrotoxicity.
\end{abstract}

Keywords: Formaldehyde, Vitamin C, liver, kidney

\section{Introduction}

Formaldehyde (HCHO) is a colorless, flammable gas with a pungent, suffocating odor. It is soluble in water, acetone, benzene, diethyl ether, chloroform and ethanol. HCHO used to make plastics and resins for the production of intermediates and for other miscellaneous uses. HCHO also used as disinfectant in many human medicines and cosmetics, as an antiseptic in veterinary drugs and biological and in fungicides, textiles and embalming fluids [1]. Exogenous $\mathrm{HCHO}$ is taken up into human body by ingestion, inhalation and dermal exposure. Inhaled $\mathrm{HCHO}$ appears to be readily absorbed by the upper respiratory tract but is not distributed throughout the body because of its rapid metabolism [2,3].

HCHO may affect the systemic cellular immunity, as well as local immunity in bronchus (BALT): (Bronchus Associated Lymphoid Tissue) [4,5]. However, chronic inhalation of HCHO causes hepatotoxicity [6] and nephrotoxicity [7].

Changes in the liver after absorption of formalin consist of mild or severe grade of cloudy swelling accompanied by vacuolation of the protoplasm, changes in the nuclei and leukocyte infiltration. Focal necrosis may result. Similar changes follow the inhalation of formaldehyde [8]. Furthermore, formalin was found to cause fatty changes through accumulation of lipid droplets in hepatocytes [9]. The toxic effects of HCHO have been reported to cause structural changes in the epithelial biliary cells and damage intrahepatic and extrahepatic biliary ducts [10]. HCHO exposure has led to disorders of oxidant and oxidant-antioxidant systems of the liver tissue and inflicted oxidative damage [11].

The injection of formalin or the inhalation of the vapors of formaldehyde produces cloudy swelling of the parenchyma of the kidney. Focal necrosis may result [8]. Furthermore, formalin was found to reveal the deposition of adipose tissue in kidney [9].

$\mathrm{HCHO}$ was found to induce oxidative stress through increasing lipid peroxidation [12], and formation of reactive oxygen species (ROS) [13], that's why it is scientifically accepted to attenuate this action by using a suitable antioxidant. Vit $\mathrm{E}$ was already used in previous study to protect liver against the effect of formaldehyde [14]. Vitamin $\mathrm{C}$ is considered as a strong antioxidant $[15,16]$ and used against several toxicants such as alcohol [17] and metals [18 ]. Some vitamins (such as vitamins A, E, and C) are known to play an important role in ameliorating the toxicity effects of reactive species generated by chemical agents in biological systems. Vitamins $\mathrm{C}$ and $\mathrm{E}$ are known to be potent antioxidants[19,20].

The aim of the present investigation was to determine and investigate the histological effect of formalin on liver and kidney of rat, and the protective role of vitamin c against the previous effect of formalin in male rat.

\section{Materials and Methods}

Male Albino Rats Were Obtained From Animal House Of Biology Department, Science College, Salahaddin-University -Erbil. Twelve Albino Male Rats (200-230gm) Were Divided Randomly Into Three Groups (4 Animals In Each) And Housed At 22 2 cwwith 12hr Light/Dark Cycle. They Were Supplied With Standard Laboratory Animal Care ;Fed Rats Diet And Tap Water Ad Libitum. The First Group Was The Control And Given Only Single Gavage Of One Ml Normal Saline, The Second Group Were Given A Daily Gavage Of $1 \mathrm{ml}$ Formaldehyde And The Third Group Were Given A Daily Gavage Of 1 Ml Of Vitamin C (1 $\mathrm{Mg} \backslash \mathrm{Kg} \backslash \mathrm{B} . \mathrm{Wt}$ ) And $1 \mathrm{Ml}$ Formaldehyde Intraperitoneal Injection. 
Paraffin Method: Liver Lobes And Kidney Pieces Were Removed And Fixed In Bouin's Fluid, Dehydrated, Cleared, Embedded In Paraffin And Cut Into 4-5 $\mu$ m Thick Section, Then Stained By Hematoxylin And Eosin [21].

\section{Results and Discussion}

Exposure to formaldehyde appears to be associated with hepatotoxicity in many species, including humans, following injection, ingestion, or inhalation. The present investigation dealt with the study of the effect of formalin exposure by gavage on the liver of rats and the protective role of vitamin $\mathrm{C}$ against this hepatotoxicity.

As shown in Fig 1-4, formalin has caused several histological and cytological alterations such as degeneration of the hepatocytes, and appearing of inflammatory leucocytes. The degeneration included shrunken and swelled appearance. The shrunken cells appeared with hypereosinophilic cytoplasm, condensed nucleus, and blebbed plasma membrane (Fig 4). These changes are similar to the apoptotic cell death [22]. Approximately similar microscopic manifestations in the liver include alterations in centrilobular vacuolization and focal cellular necrosis were noticed previously after exposing to formalin dose [23]. Inflammatory mononuclear cells were also found in the liver of formaldehyde exposed rats [12]. When given to the formalin exposed rats, vitamin $\mathrm{C}$ caused recovery of the liver structure and maintaining the normal architecture in which no inflammation, healthy hepatocytes and normal sinusoidal structure were revealed (Fig 5). HCHO disturbs the oxidant-antioxidant balance in various tissues and cause oxidative stress in parallel with tissue damage. In previous studies, increased MDA levels in the lung, liver, and testicular tissues of the rats exposed to

HCHO were reported [24-26]. In accordance with our findings, Strubelt et al. [27] have reported increased MDA levels in the liver tissues of HCHO-exposed animals. Similarly, Teng et al. [28] in their experimental study on isolated rat hepatocytes showed that $\mathrm{HCHO}$ at low concentrations leads to oxidative stress. Vitamin $\mathrm{C}$ is a potent antioxidant agent and exerts a protective effect against oxidative stress [17]. In our study, vitamin $\mathrm{C}$ was found to partially prevent the liver damage against HCHO intoxication. Considering the distinctive properties of vitamin $\mathrm{C}$ and the results of the present study, it is plausible that both its radicalscavenging and antioxidant actions are involved in preventing tissue damage.

High degenerative changes were found in the kidney of formalin treated rats in which formalin caused high inflammation degree and degenerative effect on kidney tubule cells in comparison to control group (Fig 1 $\& 2$ ), while such changes were disappeared when vitamin c was administrated to the formalin exposed rats (Fig 3).

It has been found that the main mechanism in the nephrotoxicity of formalin related to the oxidative stress caused by this compound through production of reactive oxygen species (ROS), and depletion of the antioxidant enzymes such as superoxide dismutase and glutathione peroxidase and also caused destruction of mitochondria $[11,12,13]$. Since vitamin $\mathrm{c}$ is considered as a strong antioxidant compound that can strongly scavenge free radicals especially ROS [29], it has been succeeded in playing a protective role against formalin hepatotoxicity and nephrotoxicity.

\section{Conclusion}

Certainly formaldehyde caused histological alterations in liver and kidney and in turn, vitamin C showed a protective role against this effect in rat.

\section{References}

[1]. International Agency for Research on Cancer (IARC): Some industrial chemicals and dyestuffs. IARC Monogr. Eval. Carcinog. Risk Chem. Hum., 29, 1982, 345-389.

[2]. M Casanova, H Heck, J Everitt, W Harrington and J Popp, Formaldehyde concentrations in the blood of rhesus monkeys after inhalation exposure. Food Chem. Toxicol., 26, 1988,715-716.

[3]. H Heck, M Casanova-Schmitz, P Dedd, E Schachter, T Witek, and T Tosun, Formaldehyde (CH2O) concentrations in the blood of humans and Fischer-344 rats exposed to CH2O under controlled conditions. Am. Ind. Hyg. Assoc. J., 46, 1985,1-3.

[4]. M Sandikci, U Eren, and S Kum, Effects of formaldehyde and xylene CD4 and CD8-positive T cells in bronchus associated lymphoid tissue in rats. Toxicol. Ind. Health, 23 2007a,471-477.

[5]. M Sandikci, U Eren, and S Kum, Effects of formaldehyde and xylene on alpha-naphthyl acetate esterase positive T-lymphocytes in bronchus associated lymphoid tissue and peripheral blood in rats. Rev. Med. Vet.,158, 2007b, 297-301.

[6]. E Kamata, M Nakadate, O Uchida, Y Ogawa, and S Suziki, Results of a 28 months chronic inhalation toxicity study of formaldehyde in male Fisher-344 rats. J. Toxicol. Sci., 22, 1997: 239-239.

[7]. S Al-Ghamdi, M Raftery, and M Yaqoob, Acute solvent exposure induced activation of cytochrome P450E1 causes proximal tubular cell necrosis by oxidative stress. Toxicol. In vitro, 17, 2003: 335-341.

[8]. H Martin, The toxic effects of formaldehyde and formalin. J. of Exper. Medi., 6(4-6) 1905, 487-518.

[9]. K Sadiye, M Sandikci, U Eren, and N Metin, Effects of Formaldehyde and Xylene Inhalations on Fatty Liver and Kidney in Adult and Developing Rats. J. of Animal and Veter. Advan. 9(2)2010,396-401.

[10]. M Dumont, C D'Hont, A Moreau, H Mbape, G Feldmann, and S Erlinger,.Retrograde injections of formaldehyde into the biliary tree induce alterations of biliaryepithelial function in rats. Hepatology 1996: 24: 1217-1223. 
[11]. O Strubelt, M Younes, R Pentz, W Kuhnel, Mechanistic study on formaldehyde-induced hepatotoxicity. J Toxicol Environ Health,27, 1989: 351-366.

[12]. H Pekmez, C Colakoglu, D Zorarsiz, D Kus, M Geturk, H Yilmaz, M Sarsilmaz, The Effect of Melatonin Hormone on Formaldehyde-Induced Liver Injury: A Light Microscopic and Biochemical Study, Firat Tip Dergisi, 13(2) 2008:92-97

[13]. I Zararsiz, M Sarsilmaz, U Tas, I Kus, S Meydan, and E Ozan, Protective effect of melatonin against formaldehyde-induced kidney damage in rats, Toxicol Ind Health. 23(10),2007, 573-9.

[14]. M Gulec, A Gurel, and F Armutcu, Vitamin E Protects Against Oxidative Damage Caused by Formaldehyde in the Liver and Plasma of Rats, Molecular and Cellular Biochemistry, 290,(1-2), 2006, 61-67

[15]. E Son, S Mo, D Rhee et al., Vitamin C blocks TNF-alphainduced NFkappa B activation And ICAM-1 expression in human neuroblastoma cells. Arch Pharmacol Res 27, 2004, 1073-1079.

[16]. J Ayo, N Minka, and M Mamman, Excitability scores of goats administered ascorbic acid and transported during hot-dry conditions. J Vet Sc 7, 2006,127-131.

[17]. M Sönmez, F Narin, D Akkuş, and A Türkmen, Melatonin and Vitamin C Administration Ameliorate Alcohol-Induced Oxidative Stress and eNOS Expression in Liver of Rats, Erciyes Tip Dergisi, 33(2), 2011,089-098

[18]. D Appenroth and $\mathrm{K}$ Winnefeld, Vitamin $\mathrm{E}$ and $\mathrm{C}$ in the prevention of metal nephrotoxicity in developing rats. Exp Toxicol Pathol.;50(4-6),1998,391-6.

[19]. C Whitehead, and T Keller, An update on ascorbic acid in poultry. World Poultry Sc J 59, 2003: 161-184.

[20]. R Suteu, I Altuntas, B Buyukvanli, O Akturk,H Koylu, N Delibas, The effects of diazozin on lipid peroxidation and antioxidant enzymes in rat erythrocytes: role of vitamins E and C. Toxicology and Industrial Health, 23, 2007, 13-17

[21]. J Kiernan, Histological and Histochemistry Methods. 1st ed. Pergomon press. Oxford. 1981

[22]. S Fenk and B Cookson, Apoptosis, Pyrotosis and Necrosis: Mechanistic description of dead and dying eukaryotic cells. Infect. Immuno. 73(4), 2005, 1907-1916.

[23]. J Beall, and A Ulsamer, Formaldehyde and hepatotoxicity: a review. J Toxicol Environ Health.14(1), 1984,1-21.

[24]. A Kamal, A Gomaa, M El Khafif, A Hammad, Plasma lipid peroxides among workers exposed to silica or asbestos dusts. Environ Res 1990: 49, 173-180.

[25]. I Dobrzynska, E Skrzydlewska, I Kasacka, Z Figaszewski, Protective effect of N-acetylcysteine on rat liver cell membrane during methanol intoxication. J Pharm Pharmacol 2000: 52: 547552.

[26]. M Tang, Y Xie, Y Yi, W Wang, Effects of formaldehyde on germ cells of male mice. Wei Sheng Yan Jiu 2003: 32: 544-548.

[27]. O Strubelt, M Younes, R Pentz, and W Kühnel, Mechanistic study n formaldehyde- induces hepatotoxicity. J Toxicol Environ Health. 27(3) 1989,351-66.

[28]. S Teng, K Beard, J Pourahmad, M Moridani, E Easson, R Poon, P O'Brien, The formaldehyde metabolic detoxification enzyme systems and molecular cytotoxic mechanism in isolated rat hepatocytes. Chem Biol Interact: 30;130-132(1-3) 2001, 285-96.

[29]. B Halliwell, and J Gutteridge, Free radicals and catalytic metals ions in human diseases: an overview. Meth. Enzymol. 186, 1990,185 .

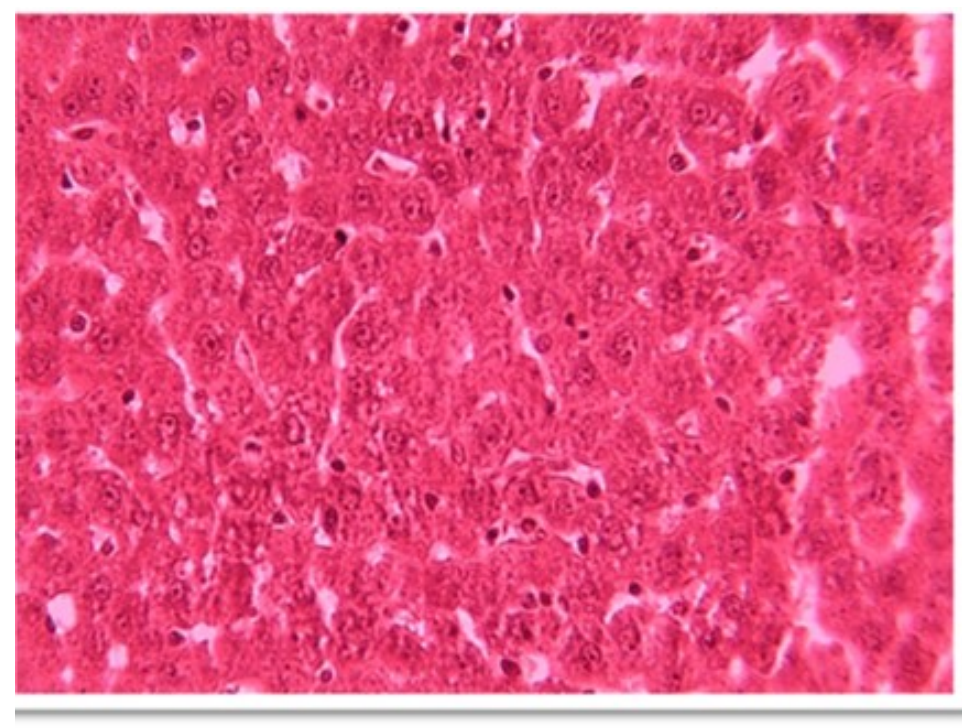

Fig (1): Control liver section showing normal histological structure, 400X. 


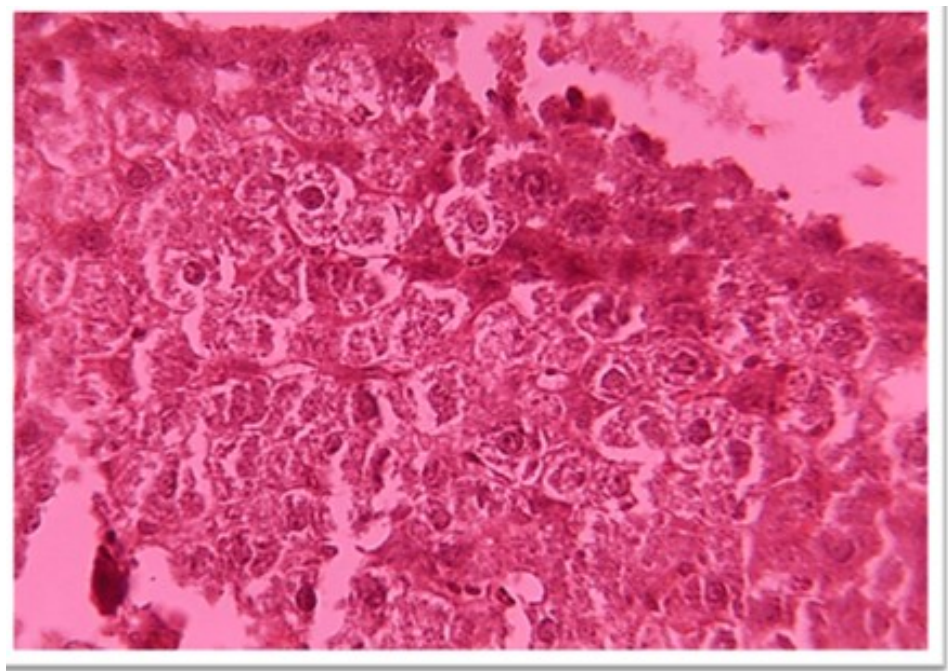

Fig (2): formalin treated rat liver showing degeneration of the liver cells, some shrunken and others are seen swelled, $400 \mathrm{X}$

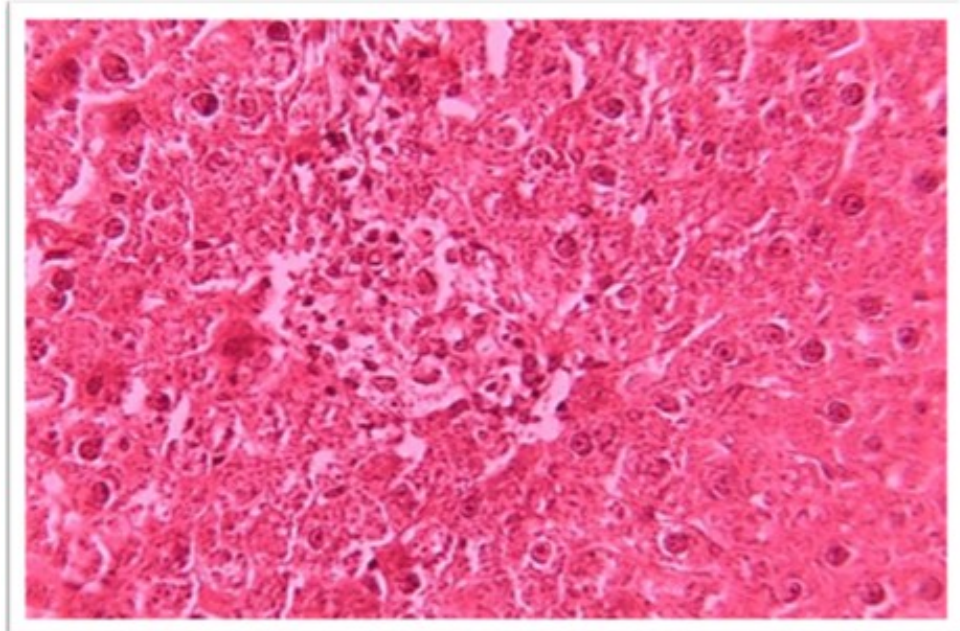

Fig (3): Formalin treated rat liver section shown accumulation of leukocytes inflammatory cells, 100x.

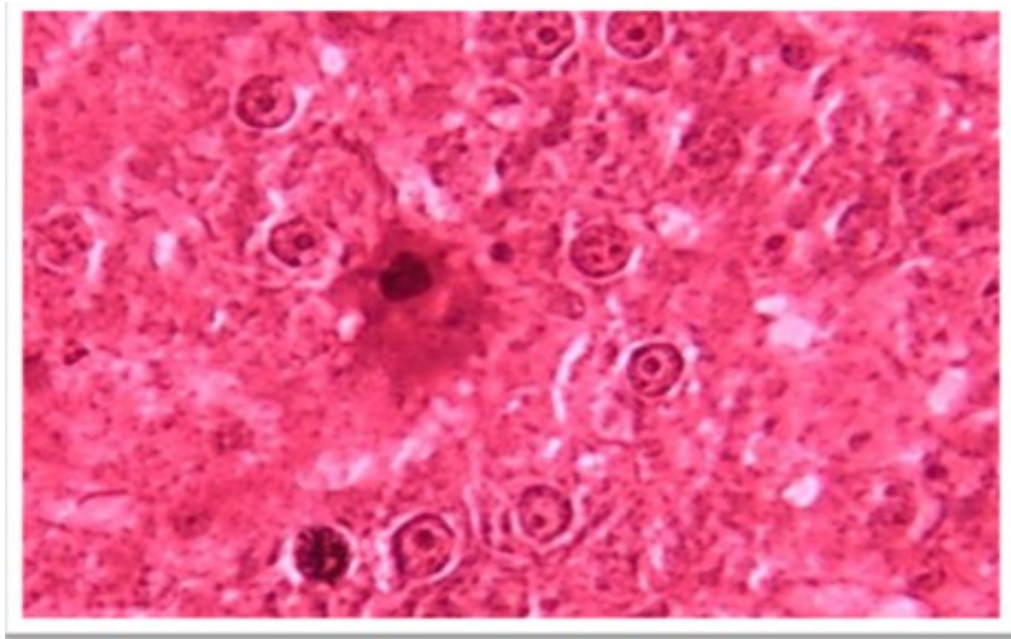

Fig (4): A typical apoptotic hepatocyte with blebbed plasma membrane, condensed nucleus and hypereosinophilic cytoplasm, 400X. 


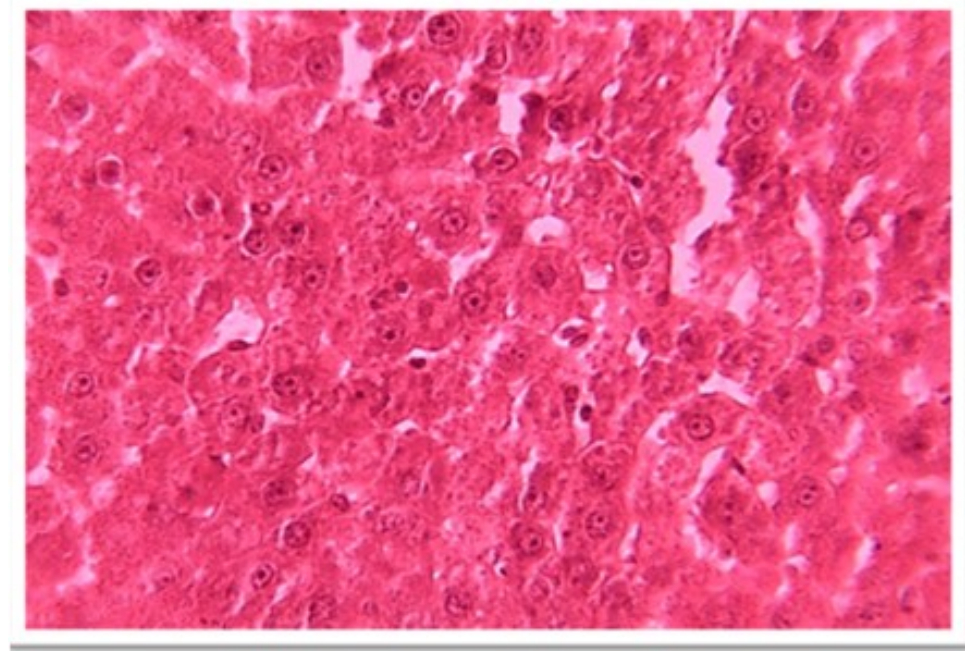

Fig(5): Section in the liver of formalin plus vitamin $C$ treated rat showing recovered structure, $400 \mathrm{X}$.

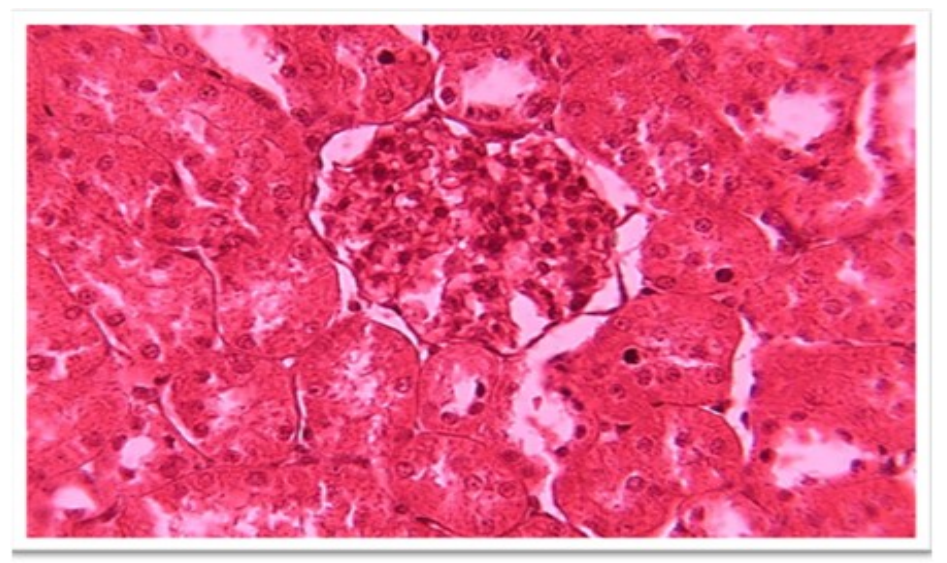

Fig(6): Sections in the kidney of control group showing healthy glomerulus and kidney tubule structure, $400 \mathrm{x}$.

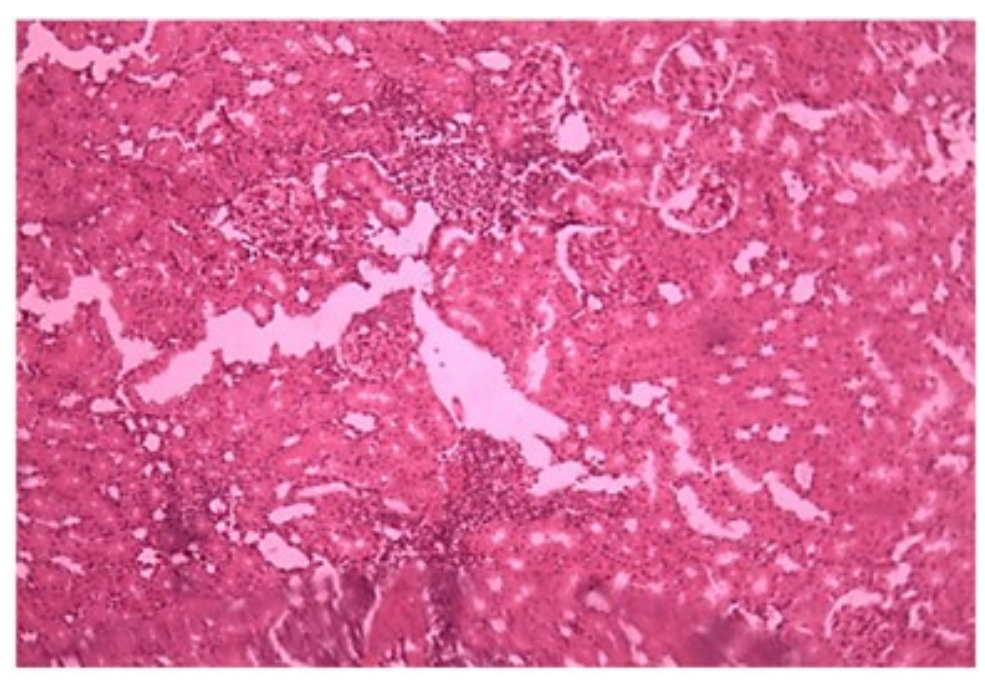




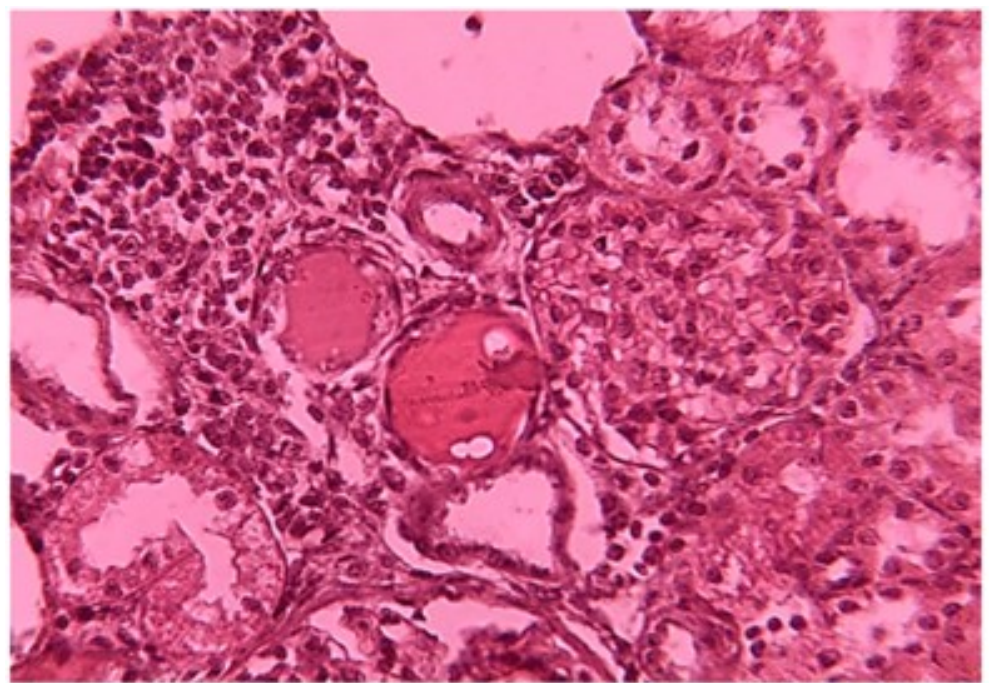

Fig (7): Sections through the kidney of formalin treated rats showing: A) inflammation (arrows) and dilation of kidney tubules (KT),100X. (above) B) Higher magnification showing the inflammatory cells and degeneration of the kidney tubule cells (arrow), 400x. (below)

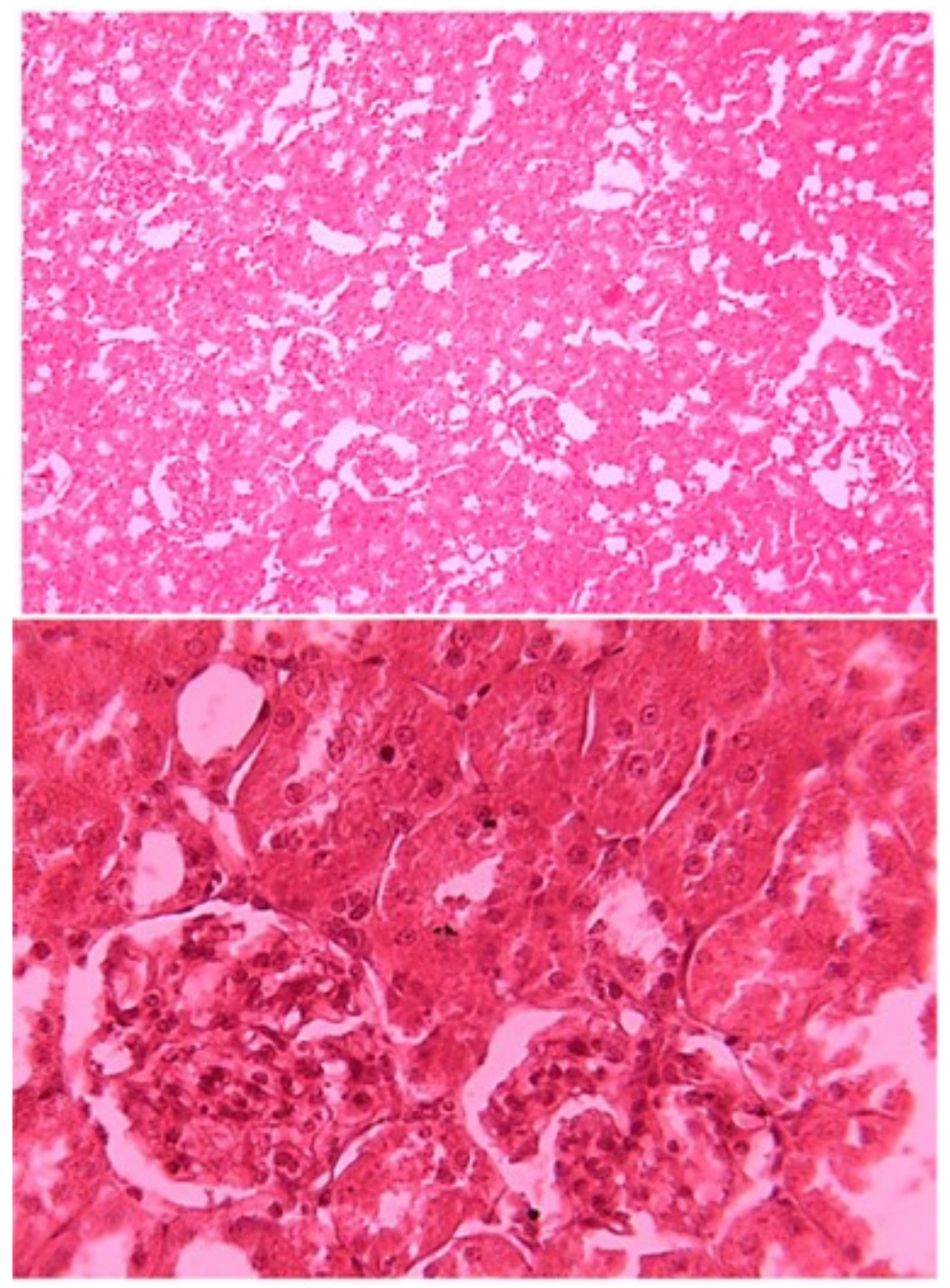

Fig (8): Sections through the kidney of formalin plus vitamin c treated rats showing approximately healthy histological structure, $100 \mathrm{X}, 400 \mathrm{x}$ respectively 